\title{
Charging non-Abelian Higgs theories
}

\author{
Oleg Antipin $\odot,{ }^{1, *}$ Jahmall Bersini $\odot,{ }^{1, \S}$ Francesco Sannino, ${ }^{2,3, \|}$ Zhi-Wei Wang $\odot^{2, *}$ and Chen Zhang $\odot^{4, \dagger}$ \\ ${ }^{1}$ Rudjer Boskovic Institute, Division of Theoretical Physics, Bijenička 54, 10000 Zagreb, Croatia \\ ${ }^{2} C P^{3}$-Origins and the Danish Institute for Advanced Study Danish IAS, University of Southern Denmark, \\ Campusvej 55, DK-5230 Odense M, Denmark \\ ${ }^{3}$ Dipartimento di Fisica "E. Pancini”, Università di Napoli Federico II-INFN sezione di Napoli \\ Complesso Universitario di Monte S. Angelo Edificio 6, via Cintia, 80126 Napoli, Italy \\ ${ }^{4}$ Physics Division, National Center for Theoretical Sciences, 300 Hsinchu, Taiwan
}

(Received 28 June 2020; accepted 30 November 2020; published 30 December 2020)

\begin{abstract}
We extend the fixed-charge semiclassical approach to compute conformal field theory (CFT) data for generic non-Abelian Higgs dynamics by also identifying the proper family of related operators. As an example, we consider the $\mathrm{U}(\mathrm{N}) \times \mathrm{U}(\mathrm{N})$ non-Abelian Higgs theory in four minus epsilon dimensions. We observe that the semiclassical method and state-operator correspondence can be used for complex CFTs, which are naturally related to walking dynamics.
\end{abstract}

DOI: $10.1103 /$ PhysRevD.102.125033

\section{INTRODUCTION}

Conformal field theories (CFTs) are of fundamental importance to our understanding of different phenomena in condensed matter [1-3], particle physics [4,5], and quantum gravity [6]. Moreover, CFT tools play a central role in unveiling the dynamics of quantum field theories (QFT) in regimes where ordinary (non)perturbative methods are either inadequate or cumbersome. The approach is to investigate the dynamics of a desired class of QFT theories by considering certain CFT limits in their parameter space. Additionally, as we shall argue below, certain theory sectors can be directly investigated using semiclassical approximations.

Here, we consider the dynamics of the non-Abelian Higgs theories because they often appear in the literature due to their relevance to particle physics and cosmology. These theories feature several marginal couplings in four dimensions that typically flow to zero at large distances and develop Landau poles at high energies, making these effective field theories. Removing the Landau poles is

\footnotetext{
*Corresponding author. wang@cp3.sdu.dk

Corresponding author.

czhang@cts.nthu.edu.tw

¥oantipin@irb.hr

\$jbersini@irb.hr

"sannino@cp3.dias.sdu.dk

Published by the American Physical Society under the terms of the Creative Commons Attribution 4.0 International license. Further distribution of this work must maintain attribution to the author(s) and the published article's title, journal citation, and DOI. Funded by SCOAP.
}

possible only if the couplings vanish along the entire renormalization group. Theories such as this one are known as trivial QFTs. One can, however, embed this type of theories into an asymptotically safe gauge-fermion-scalar theory like the one constructed in [7].

As a theoretical laboratory, we study $U(N) \times U(N)$ in $4-\epsilon$ space time dimensions. Nevertheless, our approach is broad and not limited to this example. We first show that in the infrared the model features two complex conjugated fixed points in the quartic couplings (complex CFTs) [8], signaling the appearance of a controllable near-conformal behavior of the walking type [9-14]. The latter behavior has been invoked in the literature for models of dynamical electroweak symmetry breaking [15-17]. Lattice methods have been employed to establish walking as summarized in Ref. [18]. Another way to discuss walking is via the emergence of two complex zeros of the beta function in the near-conformal phase $[9,19]$.

It is noteworthy that in the fixed-charge sector of these theories, one can obtain nontrivial information about their conformal dynamics by expanding around the fixed-charge stationary trajectories. A large-charge expansion method is initiated in $[20,21]$ and further developed in [22-25] to compute CFT operator spectrum. More recently, a semiclassical method, which can also access the small-charge regime, has been developed in [26] for the simple $\lambda(\bar{\phi} \phi)^{2}$ model in $4-\epsilon$ dimensions with a $U(1)$ global symmetry. In the small-charge regime, the method allows us to determine the scaling dimensions of a family of fixed charged operators to the leading and next to leading order terms in the charge expansion but to all-orders in the couplings. The method is more powerful compared to the conventional perturbative approach (CPA) in that it automatically 
organizes the computation of scaling dimensions for a whole infinite tower of operators rather than a single one. Moreover, it determines the terms that are difficult to obtain within CPA, therefore, significantly boosting conventional high-loop computations [27].

In this work, we generalize the semiclassical method to theories with non-Abelian symmetries beyond the $U(1)$ Abelian case [26] and non-Abelian $O(N)$ case in both $4-\epsilon$ [28] and 6- $\epsilon$ dimensions [29]. We use the $U(N) \times U(N)$ Higgs model as an example to illustrate our procedure, which by itself is more general. Note that it is trivial to identify the operator from the charge configuration of $O(N)$ model, since by a symmetry rotation, it can be simplified to a single charge problem like the trivial $U(1)$ case. Thus, our task to identify the operator will be much tougher due to the nontrivial charge configuration.

The main point of our work is to deal with non-Abelian theories with more than one coupling and that the CFT is complex with an emerging walking behavior. We highlight a few novelties as follows. For a specific charge configuration, we identify a family of corresponding operators and compute their scaling dimensions. Remarkably, we find agreement with the available results stemming from conventional perturbation theory by employing the stateoperator correspondence [30,31] at a complex CFT.

Our findings complement the nonperturbative largecharge approach employed in $[32,33]$ to investigate nearconformal dynamics. Also the first nonsupersymmetric four-dimensional application to the gauged-Yukawa version of our $U(N) \times U(N)$ model is analyzed at a large charge in [23].

The work is organized as follows. We first introduce the theory and the associated complex CFT, we then introduce the charge configurations, construct the spectrum of operators at fixed charge, and then analyze the low energy spectrum of the theory associated with the related charge configuration. Using the state-operator correspondence at the complex CFT, we determine the ground state energy and associated scaling dimensions in the charge expansion. Then, combining the semiclassical approach with ordinary perturbation theory, we reconstruct the full two loops scaling dimension for the whole family of fixed-charge operators. Remarkably, we are able to write it in a form valid for real and complex couplings, encompassing the case where both couplings are purely reals and the theory walks. Finally, we compare our choice of charge configuration to the one considered in [23].

\section{THE $U(N) \times U(N)$ HIGGS MODEL AS A COMPLEX CFT}

In Euclidean spacetime, the $U(N) \times U(N)$ linear sigma model is defined via the bare Lagrangian,

$\mathcal{L}=\operatorname{Tr}\left(\partial_{\mu} H^{\dagger} \partial^{\mu} H\right)+u_{0} \operatorname{Tr}\left(H^{\dagger} H\right)^{2}+v_{0}\left(\operatorname{Tr} H^{\dagger} H\right)^{2}$, where $H$ is a $N \times N$ matrix with complex entries, which transforms in the $(N, \bar{N})$ representation of the $U(N) \times U(N)$ symmetry and can be written in terms of $2 N^{2}$ real scalar fields,

$$
(H)_{a \alpha}=\frac{\phi+i \eta}{\sqrt{2 N_{F}}} \delta_{a \alpha}+\sum_{A=1}^{N^{2}-1}\left(h^{A}+i \pi^{A}\right) T_{a \alpha}^{A}
$$

where $T_{a \alpha}^{A}$ are the generalized Gell-Mann matrices, normalized as $\operatorname{Tr}\left(\mathrm{T}^{\mathrm{A}} \mathrm{T}^{\mathrm{B}}\right)=\frac{1}{2} \delta_{\mathrm{AB}}$. The $N$-rescaled couplings of the model are $\alpha_{h}=\frac{u N}{(4 \pi)^{2}}$ and $\alpha_{v}=\frac{v N^{2}}{(4 \pi)^{2}}$ and, in terms of these rescaled couplings, the one-loop beta functions in $4-\epsilon$ dimensions read

$$
\begin{gathered}
\beta_{\alpha_{v}}=-\epsilon \alpha_{v}+4 \alpha_{v}^{2}\left(1+\frac{4}{N^{2}}\right)+16 \alpha_{v} \alpha_{h}+12 \alpha_{h}^{2} \\
\beta_{\alpha_{h}}=-\epsilon \alpha_{h}+\frac{24}{N^{2}} \alpha_{v} \alpha_{h}+8 \alpha_{h}^{2} .
\end{gathered}
$$

The beta functions to five loops have been derived in [34]. For $\alpha_{h}=0$, Lagrangian (1) reduces to the $O\left(2 N^{2}\right)$ model with the 1-loop fixed point (FP) $\alpha_{v}^{O\left(2 N^{2}\right)}=\frac{\epsilon N^{2}}{4\left(4+N^{2}\right)}$ while for $\alpha_{h} \neq 0$, two fixed points at 1-loop emerge, and they are

$$
\begin{aligned}
& \alpha_{v}^{*}=\epsilon N^{2} \frac{9+N\left(-N \pm i \sqrt{2 N^{2}-6}\right)}{8\left(27-8 N^{2}+N^{4}\right)} \\
& \alpha_{h}^{*}=-\epsilon N \frac{5 N-N^{3} \pm 3 i \sqrt{2 N^{2}-6}}{8\left(27-8 N^{2}+N^{4}\right)} .
\end{aligned}
$$

For $N>\sqrt{3}$, they are complex thus defining two complex interacting CFT's.

To elucidate the impact of the complex CFT on the dynamics of the model, we consider the infinite $N$ limit. Here, the single-trace beta function $\beta_{\alpha_{h}}$ decouples from the double-trace one. An interacting fixed point in the infrared occurs for $\alpha_{h}^{*}=\epsilon / 8$. Substituting this value in the beta function for the double-trace operator, one notices that the double-trace beta function is positive and has a minimum near the origin controlled by $\epsilon^{2}$. Therefore, the running of $\alpha_{v}$ slows near this point; i.e., its running behavior is replaced by a walking one. One can also show that such behavior persists at finite $N$.

As we showed in [28], in the $O(N)$ model, fixed-charge operators with the lowest scaling dimension are $Q$-index traceless symmetric tensors with the classical dimension $Q$. For $Q=2$ and $N>2$ [when the representation (2) is irreducible], we obtain the decomposition of the 2-index traceless symmetric tensor as 


$$
\begin{array}{r}
\square \square_{O\left(2 N^{2}\right)}=(1, \operatorname{Adj}) \oplus(\operatorname{Adj}, 1) \oplus(\operatorname{Adj}, \operatorname{Adj}) \\
\oplus\left[\left(\square, \square^{*}\right) \oplus\left(\boxminus, \boxminus^{*}\right) \oplus \text { c.c. }\right] .
\end{array}
$$

We computed the 1-loop scaling dimensions $\Delta_{1 \text {-loop }}=$ $2+\gamma_{1-\text { loop }}$ for the operators appearing in this decomposition, which are shown in the table below [with the scaling dimensions for the representations (1, Adj) and (Adj, 1) being identical],

\begin{tabular}{lcccc}
\hline Rep. & $(\mathrm{Adj}, 1)$ & (Adj, Adj) & $(\square, \square *)$ & $\left(\boxminus, \square^{*}\right)$ \\
\hline Oper. & $\operatorname{Tr}\left[\mathrm{HT}^{\mathrm{a}} \mathrm{H}^{+}\right]$ & $\operatorname{Tr}\left[\mathrm{T}^{\mathrm{a}} \mathrm{HT}^{\mathrm{a}} \mathrm{H}^{+}\right]$ & $\operatorname{Tr}\left[\mathrm{K}^{\mathrm{i}} \mathrm{HK} \overline{\mathrm{K}}^{\mathrm{i}} \mathrm{H}\right]$ & $\operatorname{Tr}\left[\mathrm{L}^{\mathrm{i}} \mathrm{H} \bar{L}^{\mathrm{i}} \mathrm{H}\right]$ \\
\hline$\gamma_{1 \text {-loop }}$ & $\frac{4 \alpha_{v}}{N^{2}}+4 \alpha_{h}$ & $\frac{4 \alpha_{v}}{N^{2}}$ & $\frac{4 \alpha_{v}}{N^{2}}+\frac{4 \alpha_{h}}{N}$ & $\frac{4 \alpha_{v}}{N^{2}}-\frac{4 \alpha_{h}}{N}$ \\
\hline
\end{tabular}

where $K^{i}\left(\bar{K}^{i}\right)$ and $L^{i}\left(\bar{L}^{i}\right)$ are the Clebsch-Gordan coefficients for the $S U(N)$ representations $\square(\square *)$ and $\boxminus\left(\square^{*}\right)$, respectively. The $\operatorname{Tr}\left[\mathrm{T}^{\mathrm{a}} \mathrm{HT}^{\mathrm{a}} \mathrm{H}^{\dagger}\right]$ appeared earlier in [35] and will be used to test our semiclassical computation. For $\alpha_{h}=0$, the operators have the same scaling dimensions due to the enhanced $O\left(2 N^{2}\right)$ symmetry.

\section{CHARGING THE SYSTEM}

It would be desirable if we can probe the complex CFT associated with the complex fixed points by methods that go beyond the conventional perturbation theory. In this regard, the semiclassical approach delineated in [26] is well suited, which extracts the scaling dimensions of the lowestlying fixed-charge operators by virtue of the state operator correspondence [20]. We therefore perform a Weyl map to a cylinder of radius $R$ (i.e., $\mathbb{R}^{d} \rightarrow \mathbb{R} \times S^{d-1}$ ), with the cylinder action given by

$$
\begin{aligned}
\mathcal{S}_{\text {cyl }}= & \int d^{d} x \sqrt{g}\left[\operatorname{Tr}\left(\partial_{\mu} H^{\dagger} \partial^{\mu} H\right)+u_{0} \operatorname{Tr}\left(H^{\dagger} H\right)^{2}\right. \\
& \left.+v_{0}\left(\operatorname{Tr} H^{\dagger} H\right)^{2}+m^{2} \operatorname{Tr}\left(H^{\dagger} H\right)\right] .
\end{aligned}
$$

Here, $g$ denotes the metric determinant and $m^{2}=\left(\frac{d-2}{2 R}\right)^{2}$ is the coefficient of the conformal coupling required by Weyl invariance. An operator with a scaling dimension $\Delta$ in the CFT corresponds to a state with energy $E=\Delta / R$, which can be computed from a fixed-charge path integral on the cylinder [26].

Following [23], it is simplest to consider a homogeneous ground state with the ansatz (with $\tau$ being the cylinder time),

$$
H_{0}(\tau)=e^{2 i M \tau} B
$$

where $M$ and $B$ are diagonal matrices. For such solutions, the value of Cartan charges for the $S U(N)_{L} \times S U(N)_{R}$ symmetries are encoded in the traceless charge matrices,

$$
\begin{aligned}
& \mathcal{Q}_{L} \equiv-V \dot{H}_{0} H_{0}^{\dagger}=-2 i V M B^{\dagger} B, \\
& \mathcal{Q}_{R} \equiv V H_{0}^{\dagger} \dot{H}_{0}=2 i V M B^{\dagger} B,
\end{aligned}
$$

with $V=R^{d-1} \Omega_{d-1}$ being the volume of $S^{d-1}$. Note $\mathcal{Q}_{L}+\mathcal{Q}_{R}=0$ follows as a consequence of our diagonal ansatz solution, Eq. (9). The normalization of the charge matrices is chosen such that the operator $\operatorname{Tr}\left(\mathbf{e}_{21} H \mathbf{e}_{12} H^{\dagger}\right)$, with the $N \times N$ constant matrix $\mathbf{e}_{p q}$ defined by $\left(\mathbf{e}_{p q}\right)_{j k}=$ $\delta_{j p} \delta_{k q}$, corresponds to the charge configuration,

$$
\mathcal{Q}_{L, 1 / 2}=-\mathcal{Q}_{R, 1 / 2}=\operatorname{diag}\{-1 / 2,1 / 2,0, \ldots, 0\} .
$$

A general fixed-charge operator with charge configuration $\mathcal{Q}=\mathcal{Q}_{L}=-\mathcal{Q}_{R}$ can be constructed as, for example,

$$
\operatorname{Tr}\left[\Pi_{j}\left(\tau_{j} H \tau_{j}^{T} H^{\dagger}\right)^{\left|y_{j}\right|}\right] .
$$

Here, $y_{j}$ is an integer, determined by first choosing a root basis $\beta_{j}, j=1,2, \ldots, N-1$, onto which we decompose $\mathcal{Q}$ as

$$
\mathcal{Q}=\Sigma_{j=1}^{j=N-1} y_{j} \hat{h}_{\beta_{j}},
$$

where $\hat{h}_{\beta_{j}}$ 's are the roots mapped into the Cartan subalgebra of $S U(N)$ and satisfy the normalization condition $\operatorname{Tr}\left(\hat{h}_{\beta_{j}}^{2}\right)=\frac{1}{2}, \tau_{j}=\mathbf{e}_{p q}$ for some $p, q$ that depend on $j$ and should be chosen to be an element in the root subspace of $\beta_{j}$. For a given charge configuration, one may obtain variations of Eq. (12) by changing the root basis, redistributing the trace operation, or changing the order of different $\tau_{j} H \tau_{j}^{T} H^{\dagger}$ factors, giving rise to multiple operators corresponding to a given charge configuration. In any case, the operator construction involves a decomposition equation like Eq. (13). The normalization condition dictates entries of $\hat{h}_{\beta_{j}}$ 's must be integers or half-integers, and since $y_{j}$ 's must be integers [see Eq. (12)], we conclude entries of $\mathcal{Q}$ must be integers or half-integers as well.

The operator identification for a generic charge configuration is complicated and not unique. However, it can be shown that a special family of charge configurations,

$$
\mathcal{Q}_{L, J}=-\mathcal{Q}_{R, J}=\operatorname{diag}\{-\mathrm{J}, \mathrm{J}, 0, \ldots, 0\},
$$

with $J$ being a positive integer or half-integer, corresponds to a unique fixed-charge operator $\mathcal{O}_{J}=\operatorname{Tr}\left[\left(\mathbf{e}_{21} H \mathbf{e}_{12} H^{\dagger}\right)^{2 J}\right]$ with minimal classical scaling dimension $Q=4 J$ living in the representation $\left(\Gamma_{J}, \Gamma_{J}\right)$ of $S U(N)_{L} \times S U(N)_{R}$, with the irreducible representation $\Gamma_{J}$ of $S U(N)$ defined through its Dynkin label $(2 J, 0, \ldots, 0,2 J)$. For $J=1 / 2, \Gamma_{J}$ reduces to the adjoint of $S U(N)$. The uniqueness is related to the fact that this special family of charge configurations corresponds to the highest weights in the tensor product of the adjoint representations. More detail about operator 
identification from charge configurations is presented in the Appendixes.

Motivated by the form of $\mathcal{Q}_{L, J}, \mathcal{Q}_{R, J}$, we parametrize the $M, B$ matrices as $M=-i \operatorname{diag}\{\mu,-\mu, 0, \ldots, 0\}, \mathrm{B}=$ $\operatorname{diag}\{\mathrm{b}, \mathrm{b}, 0, \ldots, 0\}$, with $\mu>0, b>0$. Then according to Eq. (10), we have $J=2 V \mu b^{2}$. The ansatz Eq. (9) with this form of $M, B$ satisfies the equations of motion (EOM) derived from the cylinder action as long as

$$
2 \mu^{2}=\left(u_{0}+2 v_{0}\right) b^{2}+\frac{m^{2}}{2},
$$

which fixes $\mu$ and $b$ for given $J$ and $m$.

The fixed-charge path integral is equivalent to an unconstrained path integral with an effective action $\mathcal{S}_{\text {eff }}$ obtained by adding appropriate boundary terms resulting in adding $16 \mu^{2} b^{2}$ to $\mathcal{S}_{\text {cyl }}$ [26]. The action $\mathcal{S}_{\text {eff }}$ evaluated on the solution Eq. (9) gives classical energy. To compute the leading quantum correction, we expand $\mathcal{S}_{\text {eff }}$ around the fixed-charge solution Eq. (9) to obtain an effective Lagrangian $\mathcal{L}_{\text {quad }}$ to a quadratic order in the fluctuation field. The leading quantum correction is then computed through the functional determinant associated with $\mathcal{L}_{\text {quad }}$, which is equivalent to a sum of dispersion relations over all degrees of freedom.

The dispersion relations and their multiplicity can be explicitly worked out,

$$
\begin{aligned}
\omega_{1} & =\sqrt{J_{\ell}^{2}+4 \mu^{2}} \quad 4 N-8 \text { d.o.f. } \\
\omega_{2} & =\sqrt{J_{\ell}^{2}+4\left(1-a_{0}\right) \mu^{2}+a_{0} m^{2}} \quad 2(N-2)^{2} \text { d.o.f } \\
\omega_{3,4} & =\sqrt{J_{\ell}^{2}+4 \mu^{2}} \mp 2 \mu \quad 2 N-3 \text { d.o.f. each } \\
\omega_{5,6} & =\sqrt{J_{\ell}^{2}+4 \mu^{2}+2\left(4 \mu^{2}-m^{2}\right) a_{0}} \pm 2 \mu \quad \text { one d.o.f.each } \\
\omega_{7,8} & =\sqrt{J_{\ell}^{2}+12 \mu^{2}-m^{2} \pm \sqrt{16 \mu^{2} p^{2}+\left(m^{2}-12 \mu^{2}\right)^{2}}} \\
\omega_{9,10} & =\left(J_{\ell}^{2}+\left(8+4 a_{0}\right) \mu^{2}-a_{0} m^{2}\right. \\
& \pm \sqrt{\left.\left[\left(8+4 a_{0}\right) \mu^{2}-a_{0} m^{2}\right]^{2}+16 \mu^{2} J_{\ell}^{2}\right)^{\frac{1}{2}}}
\end{aligned}
$$

where $a_{0}=\frac{u_{0}}{u_{0}+2 v_{0}}$, and $J_{\ell}^{2}=\ell(\ell+d-2) / R^{2}$ corresponds to the eigenvalues of the Laplacian on $S^{d-1}$. Goldstone modes appear as a consequence of symmetry breaking enforced by fixing the charge. The symmetry breaking pattern can be written as $\mathcal{G}_{0} \stackrel{\text { exp }}{\rightarrow} \mathcal{G}_{1} \stackrel{\text { spont }}{\rightarrow} \mathcal{G}_{2}$, with

$$
\begin{aligned}
\mathcal{G}_{0} \equiv & S U(N)_{L} \times S U(N)_{R} \times U(1)_{A} \\
\mathcal{G}_{1} \equiv & S U(N-2)_{L} \times S U(N)_{R} \times U(1)_{L 3} \times U(1)_{L 5} \times U(1)_{A} \\
\mathcal{G}_{2} \equiv & S U(N-2)_{L} \times S U(N-2)_{R} \times U(1)_{D 3} \\
& \times U(1)_{D 5} \times U(1)_{A 6} .
\end{aligned}
$$

Here, $S U(N-2)_{L}$ denotes the left $S U(N-2)$ transformations with generators living in the lower $(N-2) \times(N-2)$ block, and the remaining $U(1)$ 's are generated from

$$
\begin{aligned}
U(1)_{L 3(D 3)} & \rightarrow \operatorname{diag}\{1,-1,0, \ldots, 0\}_{\mathrm{L}(\mathrm{D})} \\
U(1)_{L 5(D 5)} & \rightarrow \operatorname{diag}\{1,1,0, \ldots, 0\}_{\mathrm{L}(\mathrm{D})} \\
U(1)_{A 6} & \rightarrow \operatorname{diag}\{0,0,1, \ldots, 1\}_{\mathrm{A}},
\end{aligned}
$$

where the subscript L means left transformation, D means the diagonal part of left and right transformation [i.e., $U(1)_{L+R}$, and finally A means axial part of the left and right transformation [i.e., $U(1)_{L-R}$ ]. We omitted the extra vectorial $U(1)$ symmetry in the breaking pattern analysis above because it acts as a spectator.

Since Lorentz symmetry is explicitly broken, we expect both relativistic (type I) and nonrelativistic (type II) Goldstones [36]. In fact, the minus sign solution in $\omega_{3,4}$ corresponds to $2 N-3$ type II Goldstones, while two additional type I Goldstones come from the minus sign solution in $\omega_{7,8}$ and $\omega_{9,10}$, with the one in $\omega_{7,8}$ being the conformal mode. When each type I Goldstone is counted once and type II Goldstone twice, the sum of Goldstone degrees of freedom is $4 N-4$, which matches the number of broken generators at the spontaneous breaking step, saturating the Nielsen-Chadha bound [36].

\section{SEMICLASSICAL ANALYSIS AND RESULTS}

The leading order (LO) ground state energy $E_{\mathrm{LO}}$ is obtained by evaluating the effective action $\mathcal{S}_{\text {eff }}$ on the classical trajectory (9) and reads

$E_{\mathrm{LO}}=\frac{m N\left(48\left(\frac{\mu}{m}\right)^{4}-8\left(\frac{\mu}{m}\right)^{2}-1\right)}{16\left(\alpha_{h}+\alpha_{y}\right)} \quad$ and $\quad \alpha_{y} \equiv 2 \alpha_{v} / N$.

Using the EOM (15) and that $J=2 V \mu b^{2}$, we can express $\frac{\mu}{m}$ in terms of the parameter $\mathcal{J} \equiv 2 J \frac{\alpha_{h}+\alpha_{y}}{N}$ as

$$
\frac{\mu}{m}=\frac{1}{2} \frac{3^{\frac{1}{3}}+x^{\frac{2}{3}}}{3^{\frac{2}{3}} x^{\frac{1}{3}}}
$$

where $x=36 \mathcal{J}+\sqrt{-3+1296 \mathcal{J}^{2}}$. The coupling $\mathcal{J}$ controls the transition between the large charge $(\mathcal{J} \gg 1)$ and the perturbative $(\mathcal{J} \ll 1)$ regimes. Our final expression for the leading contribution to the anomalous dimension $\Delta_{\mathrm{LO}}=R E_{\mathrm{LO}}$ is

$$
\Delta_{\mathrm{LO}}=\frac{N}{144\left(\alpha_{h}+\alpha_{y}\right)}\left[\frac{3^{\frac{1}{3}}\left(3^{\frac{1}{3}}+x^{\frac{2}{3}}\right)^{4}}{x^{\frac{4}{3}}}-2 \frac{3^{\frac{2}{3}}\left(3^{\frac{1}{3}}+x^{\frac{2}{3}}\right)^{2}}{x^{\frac{2}{3}}}-9\right],
$$


and, notice that in $\Delta_{\mathrm{LO}}$, we used generic values for the couplings $\alpha_{h}$ and $\alpha_{y}$ since at LO Lagrangian (1) is Weyl invariant for any values of the couplings.

We now proceed with the computation of the leading quantum corrections $\Delta_{\mathrm{NLO}}$. Its bare expression is given by the fluctuation functional determinant and reads

$$
\Delta_{\mathrm{NLO}}^{\mathrm{bare}}=\frac{R}{2} \sum_{\ell=0}^{\infty} n_{\ell}\left[\sum_{i} g_{i}(N) \omega_{i}(\ell)\right],
$$

where $n_{\ell}=\frac{(2 \ell+d-2) \Gamma(\ell+d-2)}{\Gamma(\ell+1) \Gamma(d-1)}$ is the Laplacian multiplicity on $S^{d-1}$. The inner sum runs over all the dispersion relations $\omega_{i}$ computed in (16), each counted with its multiplicity $g_{i}(N)$.

After renormalization, we can express $\Delta_{\mathrm{NLO}}$ in terms of a convergent sum, which can be computed numerically.
Thus, following the procedure of [26], we arrive at our final expression for the NLO contribution in the semiclassical expansion at the fixed points, which reads

$$
\Delta_{\mathrm{NLO}}=\rho+\frac{1}{2} \sum_{\ell=0}^{\infty}\left[R(1+\ell)^{2}\left(\sum_{i} g_{i}(N) \omega_{i}(\ell)\right)_{d=4}+\sigma\right] .
$$

The functions $\rho\left(\mathcal{J}^{*}, N, \alpha_{h}^{*}, \alpha_{y}^{*}\right)$ and $\sigma\left(\ell, \mathcal{J}^{*}, N, \alpha_{h}^{*}, \alpha_{y}^{*}\right)$ are given in the Appendixes. Our results (23) and (25) resum to all orders in the coupling $\mathcal{J}$ the LO and NLO terms in the charge expansion, respectively. We now focus on the perturbative regime at small $\mathcal{J}$, where the sum of (23) and (25) evaluated at the FPs reads

$$
\Delta_{\mathrm{LO}}+\Delta_{\mathrm{NLO}}=Q\left[1+\frac{Q\left(\alpha_{h}^{*}+\alpha_{y}^{*}\right)}{N}-\frac{2(7+2 N) \alpha_{h}^{* 2}+(9+8 N) \alpha_{h}^{*} \alpha_{y}^{*}+\left(5+N^{2}\right) \alpha_{y}^{* 2}}{N\left(\alpha_{y}^{*}+\alpha_{h}^{*}\right)}+\mathcal{O}\left(\mathcal{J}^{2}\right)\right]
$$

where $Q=4 J$ is the classical dimension of the operator, and for the $\mathcal{O}(\mathcal{J})$ term, we substituted $\mathcal{J}=Q \frac{\alpha_{h}^{*}+\alpha_{y}^{*}}{2 N} \operatorname{explicitly.~We}$ checked that for $\alpha_{h}^{*}=0$ the above results reproduce the anomalous dimension of the $Q$-index traceless symmetric $O\left(2 N^{2}\right)$ tensor with the classical dimension $Q$. The presence of the couplings at the denominator in the perturbative expansion is a somewhat surprising feature of our results which, at first sight, can look suspicious. Nevertheless, one has to remind oneself that the above expression is strictly valid only at the fixed points so one should look at the conformal dimension $\Delta$ as a function of $\epsilon$ and not as a function of the couplings.

Considering the FPs (5), we obtain the scaling dimension at $\mathcal{O}(\epsilon)$, which reads

$$
\begin{aligned}
\Delta= & Q+\left(\frac{-126+10 N+34 N^{2}-2 N^{3}-4 N^{4} \pm i(6-2 N) \sqrt{2 N^{2}-6}}{8\left(27-8 N^{2}+N^{4}\right)} Q+\frac{18-5 N-2 N^{2}+N^{3} \pm i(2 N-3) \sqrt{2 N^{2}-6}}{8\left(27-8 N^{2}+N^{4}\right)} Q^{2}\right) \epsilon \\
& +\mathcal{O}\left(\epsilon^{2}\right) .
\end{aligned}
$$

From the group-theoretical arguments given in the previous section, this result should correspond to the 1-loop scaling dimensions for the family of the charged operators $\mathcal{O}_{J}=\operatorname{Tr}\left[\left(\mathbf{e}_{21} H \mathbf{e}_{12} H^{\dagger}\right)^{Q / 2}\right]$. It is easy to check that indeed for $Q=2$ and at 1-loop, Eq. (27) matches the anomalous dimension of the biadjoint operator $\operatorname{Tr}\left[\mathrm{T}^{\mathrm{a}} \mathrm{HT}^{\mathrm{a}} \mathrm{H}^{\dagger}\right]$ at the fixed point Eq. (5) shown in Table (7). The remarkable agreement between our results with the ones stemming from conventional perturbation theory shows that the state-operator correspondence $[30,31]$ and the semiclassical method can be applied to a complex CFT.
We can combine our semiclassical results with the knowledge of the 2-loop anomalous dimension for $\operatorname{Tr}\left[\mathrm{T}^{\mathrm{a}} \mathrm{HT}^{\mathrm{a}} \mathrm{H}^{\dagger}\right]$ [35], to extract the complete 2-loop anomalous dimension for the whole family. Remarkably, assuming an ordinary perturbative power series expansion in both couplings combined with the constraint that for $\alpha_{h}=0$, we reproduce the known result for the $O\left(2 N^{2}\right)$ model [28]. We can write it in a form valid beyond the FPs; i.e., it holds for any perturbative values of the couplings. We have

$$
\begin{aligned}
\Delta_{2-\text { loops }}= & Q+\frac{Q(Q-1) \alpha_{y}}{N}+\frac{Q(Q-2) \alpha_{h}}{N}-Q\left[2\left(\frac{3}{N^{2}}-\frac{4}{N}-1\right) \alpha_{h}^{2}+4\left(\frac{2}{N^{2}}-\frac{3}{N}\right) \alpha_{h} \alpha_{y}+\frac{1}{2}\left(\frac{1}{N^{2}}-3\right) \alpha_{y}^{2}\right] \\
& +Q^{2}\left[2\left(\frac{1}{N^{2}}-\frac{2}{N}\right) \alpha_{h}^{2}+4\left(\frac{3}{N^{2}}-\frac{2}{N}\right) \alpha_{h} \alpha_{y}+\left(\frac{3}{N^{2}}-1\right) \alpha_{y}^{2}\right]-\frac{2 Q^{3}\left(\alpha_{h}+\alpha_{y}\right)^{2}}{N^{2}}
\end{aligned}
$$


where we highlighted in red the term that was not predicted by our semiclassical result as it is an NNLO in the charge expansion. It would be interesting to extend this strategy to higher loops.

The above shows that the semiclassical approach can be successfully applied to four-dimension real theories, which describe near-conformal physics featuring complex CFTs.

Before concluding, we note that the charge configuration used so far differs from the one adopted in [23] when investigating the large charge regime of the $U(N) \times U(N)$ linear sigma model embedded in a safe theory. In their case, the charge assignment reads $\mathcal{Q}_{L, J}^{*}=-\mathcal{Q}_{R, J}^{*}=$ $\operatorname{diag}\{\underbrace{J, \ldots, J}_{\mathrm{N} / 2}, \underbrace{-J, \ldots,-J}_{\mathrm{N} / 2}\}$. For such a configuration, the classical dimension $Q$ of the corresponding fixed-charge operator satisfies $Q=2 J N$. As discussed earlier, $J$ must be positive and an integer or half-integer with the minimal value $1 / 2$; thus, in this case, $Q$ is nontrivially related to $N$ by the constraint $Q \geq N$. As a consequence, for a given $Q$, the irreducible representation to which the operator belongs depends on the value of $N$, making the identification of such operators highly nontrivial. For instance, using the semiclassical method exploited here, we have checked that for $Q=2$ and arbitrary $N>2$, the corresponding result does not match any of the operators in Table (7), while for $N=2$ and arbitrary $Q$ it coincides with Eq. (28). Notice that this is a simple consequence of the fact that for $N=2$, this charge configuration coincides with ours. The bottom line is that one needs to choose which charge configuration to consider with care when analyzing the fixed charge sectors of a CFT. We will further analyze this issue and related ones in a follow-up paper.

In summary, in this work, we generalize the semiclassical method to non-Abelian theories with nontrivial charge configurations. For the first time, we employ, with an explicit example, to illustrate that the semiclassical method and state-operator correspondence can be applied to complex CFTs.

\section{ACKNOWLEDGMENTS}

The work of O.A. and J. B. is partially supported by the Croatian Science Foundation Project No. 4418 as well as European Union through the European Regional Development Fund - the Competitiveness and Cohesion Operational Programme (Program No. KK.01.1.1.06). F. S and Z.W acknowledge the partial support by Danish National Research Foundation Grant No. DNRF:90.

\section{APPENDIX A: OPERATOR IDENTIFICATION FROM CHARGE CONFIGURATIONS}

For simplicity, we confine ourselves to charge configurations that satisfy $\mathcal{Q}_{L}+\mathcal{Q}_{R}=0$, dictated by the diagonal ansatz solution, and simply write $\mathcal{Q}_{L}$ as $\mathcal{Q}$. $\mathcal{Q}$ lives in the Cartan subalgebra $\mathcal{H}$ of $S U(N)$, and its meaning is characterized by the set of charge eigenvalues associated with a set of orthonormal basis elements in $\mathcal{H}$. Suppose $\hat{h}$ is one of the basis elements. In the self-representation of $S U(N)$ in which $\hat{h}$ is a traceless diagonal $N \times N$ matrix, we determine the proper normalization condition for $\hat{h}$ as $\operatorname{Tr}\left(\hat{h}^{2}\right)=\frac{1}{2}$. For example, $\hat{h}_{j} \equiv \frac{1}{2}\left(\mathbf{e}_{j, j}-\mathbf{e}_{j+1, j+1}\right)$ for $j=$ $1,2, \ldots, N-1$ are normalized elements (although one should be careful that for adjacent $j$ 's the elements are not orthogonal). For any normalized basis element $\hat{h}$, the associated charge eigenvalue $q_{h}$ for a given charge configuration $\mathcal{Q}$ can be computed as

$$
q_{h}=2 \operatorname{Tr}(\mathcal{Q} h) .
$$

Note that only for orthonormal basis elements this is equivalent to the coefficient extracted from the decomposition of $\mathcal{Q}$ onto basis elements, and different choices of orthonormal basis give charge eigenvalues that are compatible with each other. The key relation in fixing all these normalizations is the following commutation relation in $S U(N)$ Lie algebra:

$$
\left[\hat{h}_{j}, \mathbf{e}_{p q}\right]=\frac{1}{2}\left(\delta_{j p}-\delta_{j q}-\delta_{j+1, p}+\delta_{j+1, q}\right) \mathbf{e}_{p q},
$$

for $j=1,2, \ldots, N-1$ and $p, q=1,2, \ldots, N$ with $p \neq q$. A special case is

$$
\left[\hat{h}_{j}, \mathbf{e}_{j, j+1}\right]=\mathbf{e}_{j, j+1},
$$

when this equation is turned into a commutation relation between Noether charge and fixed-charge operators constructed from fields that satisfy canonical commutation relations. The standard normalization condition introduced above is then implied.

The general method to construct fixed-charge operator with the minimal classical scaling dimension corresponding to a given charge configuration starts with building blocks that have simple definite transformation properties under $S U(N)_{L} \times S U(N)_{R} \times U(1)_{A}$. Since we are concerned with charge configurations that satisfy $\mathcal{Q}_{L}+\mathcal{Q}_{R}=0$, the building block takes the form $\operatorname{Tr}\left(\tau H \tau^{T} H^{\dagger}\right)$ with $\tau$ being an element in some root subspace of the $S U(N)$ Lie algebra. Obviously, this object lives in the biadjoint representation of $S U(N)_{L} \times S U(N)_{R}$. To build operators with more fields, one replicates the same structure inside the same trace operation, such as

$$
\operatorname{Tr}\left[\Pi_{j}\left(\tau_{j} H \tau_{j}^{T} H^{\dagger}\right)^{\left|y_{j}\right|}\right] .
$$


Here, $y_{j}$ is an integer, and $\tau_{j}=\mathbf{e}_{p q}$ for some $p, q$ that depend on $j$. More generally, one may choose to redistribute the trace operation (i.e., splitting one single trace to multiple traces) and changing the order of matrix products for different $\tau_{j} H \tau_{j}^{T} H^{\dagger}$ factors, to obtain more operators associated with the same charge configuration. The value of $y_{j}$ should be determined from the charge configuration $\mathcal{Q}$. Since we consider operators with the minimal classical scaling dimension, it suffices to decompose $\mathcal{Q}$ onto a root basis $\beta_{j}, j=1,2, \ldots, N-1$, which in precise terms mean to consider the decomposition,

$$
\mathcal{Q}=\Sigma_{j=1}^{j=N-1} y_{j} \hat{h}_{\beta_{j}},
$$

where $\hat{h}_{\beta_{j}}$ 's are the roots mapped into $\mathcal{H}$, which satisfy the standard normalization condition $\operatorname{Tr}\left(\hat{h}_{\beta_{j}}^{2}\right)=\frac{1}{2}$. Note $\hat{h}_{\beta_{j}}$ 's are not orthogonal, and $y_{j}$ 's do not correspond to charge eigenvalues. However, by making a connection to an arbitrary orthonormal basis, one may prove that when $\tau_{j}$ in Eq. (A4) is chosen to be an element in the root subspace of $\beta_{j}, y_{j}$ should be determined from the decomposition in Eq. (A5).

For instance, for a class of charge configurations $\mathcal{Q}_{G}=\operatorname{diag}\left\{\mathrm{Q}_{1},-\mathrm{Q}_{1}, \mathrm{Q}_{3},-\mathrm{Q}_{3}, \ldots, \mathrm{Q}_{N-1},-\mathrm{Q}_{N-1}\right\}$, if we choose the root basis such that $\hat{h}_{\beta_{j}}=\hat{h}_{j}$, we find the nonzero $y_{j}$ 's are given by $y_{1}=2 Q_{1}, y_{3}=2 Q_{3}, \ldots, y_{N-1}=$ $2 Q_{N-1}$. Since $y_{j}$ 's must be an integer, this implies $Q_{1}, Q_{3}, \ldots, Q_{N-1}$ must be integers or half-integers. This is not surprising since our charge configuration corresponds to a weight of Lie algebra representation and thus can only take a discrete set of values.

The number of operators that can be constructed in this manner grows drastically if we realize that we have the freedom to change the root basis and redo the decomposition, to redistribute the trace operation, and to utilize noncommutativity of matrix products. In general, we need to change the root basis in all possible manners to find one or more optimal root basis that lead to the minimal classical scaling dimension (i.e., minimizing the sum of $\left|y_{j}\right|$ ). After the optimal root bases are found, we need to consider the above-mentioned variations that all correspond to the same charge configurations. Algebraically some of the variations may be identical. Moreover, in general, all the operators constructed in this manner only correspond to the same charge configuration, or weight; however, it is not guaranteed that they are already organized into definite irreducible representations.

Due to these algebraic complications, a complete operator identification for a generic charge configuration looks quite difficult. Nevertheless, we found a special family of charge configurations defined by $\mathcal{Q}_{L, J}=$ $-\mathcal{Q}_{R, J}=\operatorname{diag}\{-J, J, 0, \ldots, 0\}$ lead to great simplification since one can prove that it corresponds to a unique fixedcharge operator $\mathcal{O}_{J}=\operatorname{Tr}\left[\left(\mathbf{e}_{21} H \mathbf{e}_{12} H^{\dagger}\right)^{2 J}\right]$ living in the representation $\left(\Gamma_{J}, \Gamma_{J}\right)$ of $S U(N)_{L} \times S U(N)_{R}$, with the irreducible representation $\Gamma_{J}$ of $S U(N)$ defined through its Dynkin label $(2 J, 0, \ldots, 0,2 J)$. Any variations either lead to operators with larger classical scaling dimensions or lead to the same operator written in a different form. The uniqueness is related to the fact that this special family of charge configurations corresponds to highest weights in tensor product of the adjoint representations.

On the other hand, if we consider $\mathcal{Q}_{F}=\operatorname{diag}\{\mathbf{J},-\mathbf{J}$, $\mathrm{J},-\mathrm{J}, \ldots, \mathrm{J},-\mathrm{J}\}(\mathrm{J}>0)$, which is the charge configuration used in [23], then it does not correspond to a unique operator in general. Moreover, according to the decomposition in Eq. (A5), it should correspond to operators with minimal classical scaling dimension $Q=2 N J$. Since the minimal nonzero value of $J$ is $\frac{1}{2}$, it implies for this family of charge configurations $Q \geq N$. Only for $N=2, J=\frac{1}{2}, \mathcal{Q}_{F}$ reduces to $\mathcal{Q}_{1 / 2}$ which corresponds to the biadjoint of $S U(2)_{L} \times S U(2)_{R}$.

\section{APPENDIX B: THE FUNCTIONS $\rho\left(\mathcal{J}^{*}, N, \alpha_{h}^{*}, \alpha_{y}^{*}\right)$ AND $\sigma\left(\ell, \mathcal{J}^{*}, N, \alpha_{h}^{*}, \alpha_{y}^{*}\right)$}

Here, we provide explicit expressions for the functions $\rho\left(\mathcal{J}^{*}, N, \alpha_{h}^{*}, \alpha_{y}^{*}\right)$ and $\sigma\left(\ell, \mathcal{J}^{*}, N, \alpha_{h}^{*}, \alpha_{y}^{*}\right)$, which appear in our result (25) for the NLO contribution to the anomalous dimension in the semiclassical expansion.

Recalling that $\alpha_{y}^{*}=\frac{2 \alpha_{v}^{*}}{N}$ and that $x^{*}=36 \mathcal{J}^{*}+$ $\sqrt{-3+1296 \mathcal{J}^{* 2}}$, we have 


$$
\begin{aligned}
& \rho\left(\mathcal{J}^{*}, N, \alpha_{h}^{*}, \alpha_{y}^{*}\right) \\
& =\frac{1}{240\left(\alpha_{h}^{*}+\alpha_{y}^{*}\right)^{2}}\left[\frac { 1 0 } { 3 } \alpha _ { h } ^ { * 2 } \left(-72 N^{2}-\frac{323^{1 / 3} N\left(x^{* 2 / 3}+3^{1 / 3}\right)^{4}}{x^{* 4 / 3}}-\frac{483^{2 / 3} N\left(x^{* 2 / 3}+3^{1 / 3}\right)^{2}}{x^{* 2 / 3}}+162 N\right.\right. \\
& \left.-\frac{\left(43^{2 / 3} x^{* 4 / 3}+15 x^{* 2 / 3}+123^{1 / 3}\right)\left(123^{1 / 3} \alpha_{h}^{*}\left(2 \alpha_{h}^{*}+\alpha_{y}^{*}\right)+43^{2 / 3} x^{* 4 / 3} \alpha_{h}^{*}\left(2 \alpha_{h}^{*}+\alpha_{y}^{*}\right)+3 x^{* 2 / 3}\left(18 \alpha_{h}^{* 2}+5 \alpha_{h}^{*} \alpha_{y}^{*}-4 \alpha_{y}^{* 2}\right)\right)}{x^{* 4 / 3}\left(\alpha_{h}^{*}+\alpha_{y}^{*}\right)^{2}}\right) \\
& +\frac{20}{3} \alpha_{h}^{*} \alpha_{y}^{*}\left(-\frac{\left(43^{2 / 3} x^{* 4 / 3}+15 x^{* 2 / 3}+123^{1 / 3}\right)\left(123^{1 / 3} \alpha_{h}^{*}\left(2 \alpha_{h}^{*}+\alpha_{y}^{*}\right)+43^{2 / 3} x^{* 4 / 3} \alpha_{h}^{*}\left(2 \alpha_{h}^{*}+\alpha_{y}^{*}\right)+x^{* 2 / 3}\left(62 \alpha_{h}^{* 2}+31 \alpha_{h}^{*} \alpha_{y}^{*}-4 \alpha_{y}^{* 2}\right)\right)}{x^{* 4 / 3}\left(\alpha_{h}^{*}+\alpha_{y}^{*}\right)^{2}}\right. \\
& \left.-\frac{83^{2 / 3} N^{2}\left(x^{* 2 / 3}+3^{1 / 3}\right)^{2}}{x^{* 2 / 3}}-54 N^{2}-\frac{323^{1 / 3} N\left(x^{* 2 / 3}+3^{1 / 3}\right)^{4}}{x^{* 4 / 3}}-\frac{163^{2 / 3} N\left(x^{* 2 / 3}+3^{1 / 3}\right)^{2}}{x^{* 2 / 3}}+90 N\right) \\
& +\alpha_{y}^{* 2}\left(10\left(\frac{4\left(x^{* 2 / 3}+3^{1 / 3}\right)^{2}}{33^{1 / 3} x^{* 2 / 3}}-1\right)\left(-\frac{45 \alpha_{h}^{*}}{\alpha_{h}^{*}+\alpha_{y}^{*}}-\frac{9 \alpha_{h}^{* 2}}{\left(\alpha_{h}^{*}+\alpha_{y}^{*}\right)^{2}}-\frac{43^{2 / 3}\left(x^{* 2 / 3}+3^{1 / 3}\right)^{2}\left(8 \alpha_{h}^{* 2}+7 \alpha_{h}^{*} \alpha_{y}^{*}+2 \alpha_{y}^{* 2}\right)}{x^{* 2 / 3}\left(\alpha_{h}^{*}+\alpha_{y}^{*}\right)^{2}}-18\right)\right. \\
& \left.\left.+N^{2}\left(-\frac{40\left(2 x^{* 8 / 3}+33 x^{* 2 / 3}+113^{1 / 3} x^{* 2}+63^{1 / 3}\right)}{3^{2 / 3} x^{* 4 / 3}}-825\right)\right)\right]
\end{aligned}
$$

and

$$
\begin{aligned}
& \sigma\left(\ell, \mathcal{J}^{*}, N, \alpha_{h}^{*}, \alpha_{y}^{*}\right) \\
& =\frac{1}{4 \ell\left(\alpha_{h}^{*}+\alpha_{y}^{*}\right)^{2}}\left[2 \alpha _ { h } ^ { * 2 } \left(-4 \ell(\ell+1)^{3} N^{2}-2 N\left(\frac{4\left(x^{* 2 / 3}+3^{1 / 3}\right)^{2}}{33^{1 / 3} x^{* 2 / 3}}-1\right)\left((2 \ell+1)^{2}-\frac{4\left(x^{* 2 / 3}+3^{1 / 3}\right)^{2}}{33^{1 / 3} x^{* 2 / 3}}\right)+\left(43^{2 / 3} x^{* 4 / 3}+15 x^{* 2 / 3}\right.\right.\right. \\
& \left.\left.+123^{1 / 3}\right) \frac{\alpha_{h}^{*} \alpha_{y}^{*}\left(3\left(6 \ell^{2}+6 \ell+5\right) x^{* 2 / 3}+43^{2 / 3} x^{* 4 / 3}+123^{1 / 3}\right)+\left(83^{2 / 3} x^{* 4 / 3}+30 x^{* 2 / 3}+243^{1 / 3}\right) \alpha_{h}^{* 2}+18 \ell(\ell+1) x^{* 2 / 3} \alpha_{y}^{* 2}}{27 x^{* 4 / 3}\left(\alpha_{h}^{*}+\alpha_{y}^{*}\right)^{2}}\right) \\
& +4 \alpha_{h}^{*} \alpha_{y}^{*}\left(-\ell(\ell+1) N^{2}\left(4 \ell(\ell+2)+\frac{4\left(x^{* 2 / 3}+3^{1 / 3}\right)^{2}}{33^{1 / 3} x^{* 2 / 3}}+3\right)-2 N\left(\frac{4\left(x^{* 2 / 3}+3^{1 / 3}\right)^{2}}{33^{1 / 3} x^{* 2 / 3}}-1\right)\left(2 \ell(\ell+1)-\frac{4\left(x^{* 2 / 3}+3^{1 / 3}\right)^{2}}{33^{1 / 3} x^{* 2 / 3}}+1\right)\right. \\
& +\left(43^{2 / 3} x^{* 4 / 3}+15 x^{* 2 / 3}+123^{1 / 3}\right) \\
& \left.\times \frac{\alpha_{h}^{*} \alpha_{y}^{*}\left(-3\left(2 \ell^{2}+2 \ell-5\right) x^{* 2 / 3}+43^{2 / 3} x^{* 4 / 3}+123^{1 / 3}\right)+2 \alpha_{h}^{* 2}\left(-3\left(2 \ell^{2}+2 \ell-5\right) x^{* 2 / 3}+43^{2 / 3} x^{* 4 / 3}+123^{1 / 3}\right)+6 \ell(\ell+1) x^{* 2 / 3} \alpha_{y}^{* 2}}{27 x^{* 4 / 3}\left(\alpha_{h}^{*}+\alpha_{y}^{*}\right)^{2}}\right) \\
& +\alpha_{y}^{* 2}\left(2\left(\frac{4\left(x^{* 2 / 3}+3^{1 / 3}\right)^{2}}{33^{1 / 3} x^{* 2 / 3}}-1\right)\left(-3 \alpha_{h}^{*} \frac{2 \ell^{2}+2 \ell+1}{\alpha_{h}^{*}+\alpha_{y}^{*}}-\frac{3 \alpha_{h}^{* 2}}{\left(\alpha_{h}^{*}+\alpha_{y}^{*}\right)^{2}}+\frac{4\left(x^{* 2 / 3}+3^{1 / 3}\right)^{2}\left(8 \alpha_{h}^{* 2}+7 \alpha_{h}^{*} \alpha_{y}^{*}+2 \alpha_{y}^{* 2}\right)}{33^{1 / 3} x^{* 2 / 3}\left(\alpha_{h}^{*}+\alpha_{y}^{*}\right)^{2}}-2 \ell^{2}-2 \ell-2\right)\right. \\
& \left.\left.-N^{2}\left(4\left(\frac{4 \ell(\ell+1)\left(x^{* 2 / 3}+3^{1 / 3}\right)^{2}}{33^{1 / 3} x^{* 2 / 3}}+\ell(\ell+1)(2 \ell(\ell+2)+1)-\frac{4\left(x^{* 2 / 3}+3^{1 / 3}\right)^{4}}{93^{2 / 3} x^{* 4 / 3}}\right)+\frac{8\left(x^{* 2 / 3}+3^{1 / 3}\right)^{2}}{33^{1 / 3} x^{* 2 / 3}}-1\right)\right)\right] \text {. }
\end{aligned}
$$

[1] K. G. Wilson and J. B. Kogut, Phys. Rep. 12, 75 (1974).

[2] P. Christe and M. Henkel, Lect. Notes Phys., M: Monogr. 16, 1 (1993).

[3] H. Kleinert and V. Schulte-Frohlinde, Critical Properties of phi **4-Theories (World Scientific, Singapore, 2001).

[4] F. Sannino, Acta Phys. Pol. B 40, 3533 (2009).

[5] V. A. Miransky and K. Yamawaki, Phys. Rev. D 55, 5051 (1997).
[6] J. M. Maldacena, Int. J. Theor. Phys. 38, 1113 (1999); Adv. Theor. Math. Phys. 2, 231 (1998).

[7] D. F. Litim and F. Sannino, J. High Energy Phys. 12 (2014) 178.

[8] A. Paterson, Nucl. Phys. B190, 188 (1981).

[9] V. Gorbenko, S. Rychkov, and B. Zan, J. High Energy Phys. 10 (2018) 108.

[10] V. Gorbenko, S. Rychkov, and B. Zan, SciPost Phys. 5, 050 (2018). 
[11] D. B. Kaplan, J. W. Lee, D. T. Son, and M. A. Stephanov, Phys. Rev. D 80, 125005 (2009).

[12] F. F. Hansen, T. Janowski, K. Langæble, R. B. Mann, F. Sannino, T. G. Steele, and Z. W. Wang, Phys. Rev. D 97, 065014 (2018).

[13] F. Benini, C. Iossa, and M. Serone, Phys. Rev. Lett. 124, 051602 (2020).

[14] A. F. Faedo, C. Hoyos, D. Mateos, and J. G. Subils, Phys. Rev. Lett. 124, 161601 (2020).

[15] B. Holdom, Phys. Rev. D 24, 1441 (1981).

[16] B. Holdom, Phys. Lett. B 213, 365 (1988).

[17] B. Holdom, Phys. Rev. Lett. 62, 997 (1989).

[18] G. Cacciapaglia, C. Pica, and F. Sannino, Phys. Rep. 877, 1 (2020).

[19] F. Sannino, Mod. Phys. Lett. A 28, 1350127 (2013).

[20] S. Hellerman, D. Orlando, S. Reffert, and M. Watanabe, J. High Energy Phys. 12 (2015) 071.

[21] L. Alvarez-Gaume, O. Loukas, D. Orlando, and S. Reffert, J. High Energy Phys. 04 (2017) 059.

[22] A. Monin, D. Pirtskhalava, R. Rattazzi, and F. K. Seibold, J. High Energy Phys. 06 (2017) 011.

[23] D. Orlando, S. Reffert, and F. Sannino, J. High Energy Phys. 08 (2019) 164.
[24] L. Alvarez-Gaume, D. Orlando, and S. Reffert, J. High Energy Phys. 12 (2019) 142.

[25] G. Arias-Tamargo, D. Rodriguez-Gomez, and J. Russo, J. High Energy Phys. 10 (2019) 201.

[26] G. Badel, G. Cuomo, A. Monin, and R. Rattazzi, J. High Energy Phys. 11 (2019) 110.

[27] I. Jack and D. R. T. Jones, Phys. Rev. D 102, 085012 (2020).

[28] O. Antipin, J. Bersini, F. Sannino, Z. W. Wang, and C. Zhang, Phys. Rev. D 102, 045011 (2020).

[29] G. Arias-Tamargo, D. Rodriguez-Gomez, and J. G. Russo, J. High Energy Phys. 09 (2020) 064.

[30] J. L. Cardy, J. Phys. A 17, L385 (1984).

[31] J. Cardy, J. Phys. A 18, L757 (1985).

[32] D. Orlando, S. Reffert, and F. Sannino, arXiv:2003.08396.

[33] D. Orlando, S. Reffert, and F. Sannino, Phys. Rev. D 101, 065018 (2020).

[34] P. Calabrese and P. Parruccini, J. High Energy Phys. 05 (2004) 018.

[35] O. Antipin, E. Mølgaard, and F. Sannino, J. High Energy Phys. 06 (2015) 030.

[36] H. B. Nielsen and S. Chadha, Nucl. Phys. B105, 445 (1976). 